BULL. AUSTRAL. MATH. SOC.

\title{
ASYMPTOTIC LINEARITY OF SOLUTIONS \\ OF NONLINEAR DIFFERENTIAL EQUATIONS
}

\author{
ShaOzhu CheN
}

In this paper we establish sufficient or necessary conditions for the nonlinear differential equation $u^{\prime \prime}+f(t, u)=0$ to have solutions which are asymptotic to lines with non-zero slopes and correct some formulations in theorems obtained by D.S. Cohen and $\mathrm{J}$. Tong.

Condiser the nonlinear differential equation

$$
u^{\prime \prime}+f(t, u)=0 \text {, }
$$

where $f(t, u)$ is a continuous function on $D: t \geq 0, u \in R=(-\infty, \infty)$. Using Bellman's method [1,pp.114-115] and Gronwall's inequality,

Cohen [3] obtained the following result:

PROPOSITION A. Suppose $f(t, u)$ satisfies the following hypotheses:

(H1) the derivative $f_{u}(t, u)$ exists and $f_{u}(t, u)>0$ on $D_{\text {; }}$

(H2) $|f(t, u)| \leq f_{u}(t, 0)|u|$ on $D$.

If in addition

Received 8 April 1986. It is a pleasure to thank Professor F.V. Atkinson for several comments and fruitful discussions.

Copyright Clearance Centre, Inc. Serial-fee code: 0004-9729/87 $\$ A 2.00+0.00$. 


$$
\int^{\infty} t f_{u}(t, 0) d t<\infty,
$$

then Equation ( 1 ) has solutions which are asymptotic to $a+b t$ as $t \rightarrow \infty$, where $a$ and $b$ are constants and $b \neq 0$.

Tong [5, Theorem $B]$ employed the same method and Bihari's inequality [2] to generalize Cohen's result:

PROPOSITION B. Suppose there are nonnegative continuous functions $v(t)$ for $t \geq 0$ and $g(u)$ for $u \geq 0$ such that

(H3) $g(u)$ is positive and nondecreasing for $u>0$; (H4) $|f(t, u)| \leq v(t) g(|u| / t)$ for $t>0$ and $u \in R$.

If in addition

$$
\int^{\infty} v(t) d t<\infty
$$

then Equation (1) has solutions which are asymptotic to $a+b t$ with $b \neq 0$ as $t \rightarrow \infty$. (Note that we have combined the $v(t)$ and $\phi(t)$ of Tong's theorem).

But both of them have proved only that Equation (1) has solutions satisfying

$$
\lim _{t \rightarrow \infty} u^{\prime}(t)=b \neq 0
$$

or

$$
\lim _{t \rightarrow \infty} \frac{u(t)}{t}=b \neq 0
$$

In general, a solution $u(t)$ is said to be asymptotic to $a+b t$ as $t \rightarrow \infty$ if

$$
u(t)=a+b t+o(1) \text { as } t \rightarrow \infty \text {. }
$$

Obviously, neither (4) nor (5) implies (6). In fact, we have the following example.

EXAMPLE 1. The linear equation

$$
u^{\prime \prime}+\frac{1}{4 t^{2}(\sqrt{t}+1)} u=0
$$

has two linearly independent solutions $u_{1}=t+\sqrt{t}$ and $u_{2}=$ $(t+\sqrt{t}) \int_{t}^{\infty} d s /(s+\sqrt{s})^{2}$. It is easy to verify that all the conditions 
of Propositions $A$ and $B$ are satisfied. However, for any unbounded solution $u(t)=C_{1} u_{1}(t)+C_{2} u_{2}(t)$ with $C_{1} \neq 0$, since $u_{2}(t)=1+o(1)$ as $t \rightarrow \infty$, we have

$$
u(t)=C_{1} t+C_{2}+C_{1} \sqrt{t}+o(1) \text { as } t \rightarrow \infty .
$$

Therefore, $u(t)$ is not asymptotic to any line $a+b t$.

The above example shows that Condition (2) (Condition (3)) is not sufficient for the conclusion in Proposition A (Proposition B) to hold, and we have to classify the solutions of (1) with the different asymptotic behaviour described by (5) and (6). This is our motivation to establish the following theorem.

THEOREM 1. Suppose Conditions (H3), (H4) and (3) are valid. Then Equation (1) has a solution satisfying (4) or, equivalently, (5). If in addition

$$
\int^{\infty} t v(t) d t<\infty,
$$

then for any $a, b \in R, b \neq 0$, Equation (1) has a solution $u(t)$ which is asymptotic to the line $a+b t$ as $t \rightarrow \infty$ and satisfies $u^{\prime}-b \in L(0, \infty)$.

Remark. The first part of Theorem 1 was proved by Tong, but the proof was imperfect since it was based on a false argument: If $c_{2}>0$ is sufficiently large, then $u^{\prime}(t) \geq 1$ for all $t \geq 1$, where $u(t)$ is the solution of (1) with $u(1)=c_{1}$ and $u^{\prime}(1)=c_{2}$. In fact, the choice of $c_{2}$ affects the choice of $c_{4}$ in the last lines of the proof (see $[5, p$ 236]).

Proof of Theorem 1. Let $c_{1}$ and $c_{2}$ be two positive numbers, $G(x)=\int_{1}^{x} d s / g(s)$ and $G^{-1}(x)$ be the inverse function of $G(x)$. According to (3), we can choose $T \geq 0$ so large that $c_{2} T>c_{1}$,

$$
G^{-1}\left(G\left(c_{2}\right)+\int_{T}^{\infty} v(s) d s\right) \leq G^{-1}\left(G\left(c_{2}\right)\right)+1=c_{2}+1
$$

and 
(10)

$$
g\left(c_{2}+1\right) \int_{T}^{\infty} v(s) d s \leq \frac{1}{2} c_{2} .
$$

Let $u(t)$ be the solution of (1) with $u(T)=c_{1}$ and $u^{\prime}(T)=c_{2}$. Then for $t \geq T$ we have

$$
u(t)=c_{1}+c_{2}(t-T)-\int_{T}^{t}(t-s) f(s, u(s)) d s
$$

and

(11)

$$
\frac{|u(t)|}{t} \leq \frac{c_{1}}{t}+c_{2}\left(1-\frac{T}{t}\right)+\int_{T}^{t}\left(1-\frac{s}{t}\right)|f(s, u(s))| d s
$$

$$
\leq c_{2}+\int_{T}^{t} v(s) g\left(\frac{|u(s)|}{s}\right) d s \text {. }
$$

Applying Bihari's inequality to (11), from (9) we obtain

$$
\frac{|u(t)|}{t} \leq G^{-1}\left(G\left(c_{2}\right)+\int_{T}^{t} v(s) d s\right) \leq c_{2}+1 .
$$

On the other hand, since

$$
\begin{aligned}
\int_{T}^{\infty}|f(s, u(s))| d s & \leq \int_{T}^{\infty} v(s) g\left(\frac{|u(s)|}{s}\right) d s \\
& \leq g\left(c_{2}+1\right) \int_{T}^{\infty} v(s) d s<\infty
\end{aligned}
$$

and

$$
\begin{aligned}
u^{\prime}(t) & =c_{2}-\int_{T}^{t} f(s, u(s)) d s \\
& \geq c_{2}-g\left(c_{2}+1\right) \int_{T}^{t} v(s) d s \geq \frac{2}{2} c_{2}>0,
\end{aligned}
$$

(4) holds for some $b>0$. Hence,

$$
u^{\prime}(t)=b+\int_{t}^{\infty} f(s, u(s)) d s
$$

and

$$
u(t)=c_{1}+b(t-T)+\int_{T}^{t} d r \int_{r}^{\infty} f(s, u(s)) d s
$$


Then (5) follows from (14) and (13) immediately. If (5) holds, then $\frac{|u(t)|}{t}$ is bounded. From the above argument the limit of $u^{\prime}(t)$ as $t \rightarrow \infty$ exists and must be equal to $b$. Therefore, (4) and (5) are actually equivalent. The proof of the first part is complete.

Assume (8) is satisfied. For any constants $a$ and $b \neq 0$, we choose a constant $T \geq 1+|a|$ so large that

$$
g(|b|+1) \int_{T}^{\infty} t v(t) d t<1 .
$$

Define a sequence $\left\{u_{n}(t)\right\}$ of continuous functions of $[T, \infty)$ as follows:

$$
\begin{aligned}
u_{n}(t) & =a+b t-\int_{t}^{T+n}(s-t) f\left(s, u_{n}(s)\right) d s \text { for } T \leq t \leq T+n, \\
& =a+b t \quad \text { for } t>T+n, \quad n=1,2, \ldots .
\end{aligned}
$$

If we set

$$
\begin{aligned}
K_{n}(s, t) & =s-t & & \text { for } \quad T \leq t \leq s \leq T+n, \\
& =0, & & \text { otherwise, }
\end{aligned}
$$

then (16) can be reduced to

$$
u_{n}(t)=a+b t-\int_{T}^{\infty} K_{n}(s, t) f\left(s, u_{n}(s)\right) d s \text { for } t \geq T, n=1,2, \ldots .
$$

From (15), it is easy to show that $\left|u_{n}(t)-a-b t\right|<1$ and $\left|u_{n}^{\prime}(t)-b\right|$ $<1$ for all $t \geq T$ and $n=1,2, \ldots$. Therefore, there is a subsequence $\left\{u_{n_{j}}(t)\right\}, j=1,2, \ldots$, which converges uniformly to a continuous function $u(t)$ on any compact subinterval in $[T, \infty)$. Moreover, for any $j=1,2, \ldots$,

$$
\left|k_{n_{j}}(s, t) f\left(s, u_{n_{j}}(s)\right)\right| \leq s v(s) g(|b|+1) .
$$

substitute $u_{n_{j}}(t)$ into (17) and let $j \rightarrow \infty ;$ then (8) implies that the dominated convergence theorem due to Lebesgue may be applied to give

$$
u(t)=a+b t-\int_{t}^{\infty}(s-t) f(s, u(s)) d s .
$$

So, $u(t)$ is a solution of (1) which is asymptotic to $a+b t$ as $t \rightarrow \infty$. 
Finally, from (18) and $\frac{|u(t)|}{t}<|b|+\frac{|a|+1}{t} \leq|b|+1$, we have

$$
\begin{aligned}
\int_{T}^{t}\left|u^{\prime}(s)-b\right| d s & =\int_{T}^{t}\left|\int_{s}^{\infty} f(r, u(r)) d r\right| d s \\
& \leq \int_{T}^{t} \int_{s}^{\infty} v(r) g\left(\frac{|u(r)|}{r}\right) d r d s \\
& \leq g(|b|+1) \int_{T}^{\infty}(s-T) v(s) d s,
\end{aligned}
$$

hence, $u^{\prime}-b \in L(0, \infty)$. This completes the proof of the theorem.

We can even find a necessary and sufficient condition for Equation

(1) to have a solution which is asymptotic to a line with non-zero slope, if one of the following hypotheses is imposed on the function $f$.

(H5) For each fixed $t \geq 0, f(t, u)$ is nondecreasing in $u$ and $u f(t, u) \geq 0$ on $D$.

(H6) For each fixed $t \geq 0, f(t, u)$ is nonincreasing in $u$ and $u f(t, u) \leq 0$ on $D$.

THEOREM 2. Suppose that (HS) of (H6) is valid.

(i) There exists a solution $u(t)$ of Equation (1) satisfying (4), if and only if, for some constant $k \neq 0$,

$$
\int^{\infty}|f(t, k t)| d t<\infty \text {. }
$$

(ii) There exists a solution $u(t)$ of (1) which is asymptotic to $a+b t$ with $b \neq 0$ as $t \rightarrow \infty$, if and only if, for some $k \neq 0$

$$
\int^{\infty} t|f(t, k t)| d t<\infty \text {. }
$$

Proof. It suffices to show the theorem under (H5), since a parallel argument may handle the case in which (H6) holds.

(i) Assume $u^{\prime}(t) \rightarrow b>0$ as $t \rightarrow \infty$ and $u(t) \geq \frac{1}{2} b t$ for $t \geq T \geq 0$. By (13) and (H5), (19) follows immediately for $k=\frac{3}{2} b$. suppose (19) holds for some $k>0$. Select a $T \geq 0$ large so that

$$
\int_{T}^{\infty}|f(t, k t)| d t<\frac{1}{2} k,
$$


let $u(t)$ be a solution of (1) with $u(T)=0, u^{\prime}(T)=k$. It is easy to see that $0<u(t)<k t$ and $\frac{z}{2} k<u^{\prime}(t)<k$ for all $t>T$. Thus $u^{\prime}(t) \rightarrow b>\frac{1}{2} k$ as $t \rightarrow \infty$. The proof is similar in the case $b<0$ and $k<0$.

(ii) Necessity. Without loss of generality, we may suppose $b>0$. Let $T \geq 0$ be so large that $u(t) \geq \frac{1}{2} b t$ for $t \geq T$. Set $w(t)=$ $a+b t-u(t)$; then $w(t) \geq 0, w^{\prime}(t)=b-u^{\prime}(t) \leq 0$ and $w(t) \rightarrow 0$ as $t \rightarrow \infty$. From (13) we have

$$
\begin{aligned}
\int_{T}^{t}(s-T) f(s, u(s)) d s & =w(T)-w(t)-\int_{t}^{\infty}(t-T) f(s, u(s)) d s \\
& \leq w(T)-w(t)
\end{aligned}
$$

and

$$
\int^{\infty} t f(t, u(t)) d t<\infty
$$

Consequently, (H5) implies that (20) holds for $k=\frac{3}{b} b$.

sufficiency. Without any loss, we suppose $k>0$. For any $0<b<k$ and $a \in R$, or $b=k$ and $a \leq 0$, we can find $a T>0$ such that

$$
0<a+b t \leq k t \text { for } t \geq T \text {. }
$$

Then, by a process similar to that one used in the proof of Theorem 1 , we can get a solution $u(t)$ of ( 1 ) which is asymptotic to $a+b t$ as $t+\infty$. Here, we prove a little more than the theorem requests.

The proof of Theorem 2 is complete.

EXAMPLE 2. BY Theorem 1, the equation

$$
u^{\prime \prime}+t^{-4} u^{2} \operatorname{sgn} u=0
$$

has solutions satisfying (4) or (5), however, according to Theorem 2, it has no solution which can be asymptotic to any line $a+b t$ with $b \neq 0$.

EXAMPLE 3.

$$
u^{\prime \prime}+\frac{1}{t^{n} \log ^{m} t} u \log (1+|u|)=0, \quad t>1 \text {. }
$$

By Theorem 2, Equation (22) has solutions satisfying (4) for $n>2$, or $n=2$ and $m>2$. Since $(20)$ is valid for any $k \neq 0$ if $n>3$, or 
$n=3$ and $m>2$, (22) has a solution asymptotic to $a+b t$ as $t \rightarrow \infty$ for any $a \in R$ and $b \neq 0$. Taking $v(t)=t^{2-n} \log ^{-m} t, g(u)=u^{2}$, however, we may use Theorem 1 to obtain only stronger conditions: $n>3$, or $n=3$ and $m>1$ for solutions satisfying (4), and $n>4$, or $n=4$ and $m>1$ for solutions asymptotic to lines with non-zero slopes to exit, since no appropriate function $g(u)$ is available to get better results.

For the linear equation

$$
u^{\prime \prime}+v(t) u=0,
$$

where $v(t)$ is a continuous function for $t \geq 0$ and either $v(t) \geq 0$ or $\dot{v}(t) \leq 0$, Theorem 2 produces the following result established by Hille [4, Theorem 3, p. 239] and stated here in a slightly different way.

COROLLARY. For ony constonts $a$ and $b$, Equation (23) has $a$ solution $u(t)=a+b t+o(1)$ as $t+\infty$, if and only if

$$
\int^{\infty} t^{2}|v(t)| d t<\infty \text {. }
$$

For example the linear equations

$$
u^{\prime \prime} \pm \frac{1}{t^{m}} u=0
$$

have solutions which are asymptotic to non-zero-slope lines if and only if $m>3$.

\section{References}

[1] R. Bellman, Stability theory of differential equations, McGraw-Hill, New York, 1953.

[2] I. Bihari, "A generalization of a lemma of Bellman and its application to uniqueness problems of differential equations", Acta Math. Acad. Sci. Hungar., 7 (1957), 81-94.

[3] D.S. Cohen, "The asymptotic behaviour of a class of nonlinear differential equations", Proc.Amer.Math.Soc., 18 (1967), 607-609.

[4] E. Hille, "Nonoscillation theorems", Trans.Amer.Math.Soc., 64 (1948), 234-252. 
[5] J. Tong, "The asymptotic behaviour of a class of nonlinear differential equations of second order", Proc.Amer.Math.Soc., 84 (1982), 235-236.

Department of Mathematics

University of Toronto

Toronto, Ontario

Canada M5S IAl.

Permanent Address

Department of Mathematics

Shandong University

Jinan, Shandong,

People's Republic of China 